\title{
CRISIS Y REESTRUCTURACIÓN EN EL ÁREA METROPOLITANA DE VALENCIA $^{1}$ \\ CRISIS AND RESTRUCTURATION IN THE VALENCIAN METROPOLITAN AREA
}

\author{
Julia Salom Carrasco \\ Universidad de Valencia \\ julia.salom@uv.es \\ Juan Miguel Albertos Puebla \\ Universidad de Valencia \\ juan.m.albertos@uv.es \\ Felix Fajardo Magraner \\ Universidad de Valencia \\ fefama@alumni.uv.es
}

Recibido: septiembre, 2014.

Versión final aceptada: abril, 2015.

PALABRAS CLAVE: Estructuras metropolitanas, industria, crisis, Área Metropolitana de Valencia.

KEY WORDS: Metropolitan structures, industry, crisis, Valencian Metropolitan Are.

\section{RESUMEN}

En el presente artículo presentamos los resultados de un análisis de la estructura socioeconómica del Área Metropolitana de Valencia en 2011 que, en comparación a la existente en 2001, permite apreciar las tendencias recientes de reestructuración territorial. A continuación, se utiliza la tipología territorial hallada como patrón para el análisis de la dinámica reciente de la inversión industrial, una de las variables económicas que ha jugado un papel más importante en la formación del área metropolitana, y que desde distintas instancias viene siendo señalada como un factor estratégico de la recuperación económica. El análisis realizado muestra que, tanto en relación con la actividad industrial como con respecto a otras variables socioeconómicas, se está produciendo una diferenciación territorial de carácter funcional cada vez más marcada, que presenta también rasgos de segregación socioeconómica. En el ámbito industrial, la recuperación de las inversiones en los años

\footnotetext{
${ }^{1}$ El presente artículo se inscribe en el marco del proyecto "Sostenibilidad y Competitividad Urbanas en un contexto global. El Área Metropolitana de Valencia" (CSO2013-46863-C3-1-R), financiado por la Secretaría de Estado de Investigación, Desarrollo e Innovación del Ministerio de Economía y Competitividad, dentro del Programa Estatal de Investigación, Desarrollo e Innovación Orientada a los Retos de la Sociedad.
} 
2011-2013 presenta rasgos de una notable polarización y concentración tanto espacial como sectorial y empresarial, lo que condiciona tanto las posibilidades de recuperación económica como el modelo territorial futuro del espacio metropolitano.

\section{ABSTRACT}

In this paper we present the results of an analysis of the changing socio-economic structure of the Metropolitan Area Valencia between 2001 and 2011, unveiling trends of recent territorial restructuring. Then, using the spatial typology found in this territorial analysis, we study the recent dynamics of industrial investment, one of the most important economic variables for the formation of the metropolitan area and often considered as a strategic factor in economic recovery. The analysis shows how an increasingly territorial and functional differentiation process is taking place leading to higher levels of economic segregation. The industrial investment recovery recorded in the years 2011-2013 is taking place with remarkable concentration patterns (at spatial, sectorial and firm level); this high polarization processes are probably affecting both the chances of economic recovery and the territorial future of the metropolitan spatial model. 


\section{INTRODUCCIÓN.}

La Comunidad Valenciana es una de las regiones españolas que ha experimentado con más fuerza el impacto del ciclo económico negativo que se inicia en España a partir del año 2006, y que se acelera a partir del año 2009 hasta concluir en la actual fase de recesión económica. La crisis económica que siguió al estallido de la burbuja inmobiliaria y financiera en 2008 ha tenido un impacto espacialmente intenso en la región, con caídas media anual del PIB del 2,3\% frente al 1,3\% del conjunto de España. El diferencial negativo se ha ido agudizando progresivamente, en particular desde 2009, de tal forma que el PIB por habitante regional se ha situado por debajo de la media nacional. No es de extrañar por tanto que el Área Metropolitana de Valencia (AMV), que constituye algo más de una tercera parte de la población y de la actividad económica regional, sea uno de los espacios urbanos españoles que han sufrido un mayor retroceso en los últimos años.

La evolución del paro registrado es un expresivo indicador de este impacto. Tal y como puede verse en el cuadro 1, durante el periodo 2001-2006 el AMV había mantenido una evolución del mercado de trabajo más favorable que la media regional; a lo largo de este periodo, el aumento de la tasa de paro es de sólo un 1,75\%, mientras que para el conjunto de la Comunidad Valenciana este índice alcanza el 7,24\%. El ritmo de incremento se mantiene por debajo de la media regional durante el periodo 20062008; al parecer, las economías propias de la dinámica metropolitana mantienen la tasa de paro por debajo de la media regional durante los primeros meses de la crisis. Sin embargo, a partir de esta última fecha, la tasa media de crecimiento anual del paro registrado en el AMV supera por primera vez en el siglo la media de la Comunidad Valenciana, alcanzando el $34,4 \%$ de incremento frente al $32,5 \%$ del conjunto regional, lo que supone la inversión de una tendencia que se había mantenido durante décadas. El deterioro del mercado de trabajo ha sido importante en todos los sectores económicos; como puede verse en el cuadro 2, aunque el crecimiento más fuerte del paro ha ocurrido en el sector de la construcción seguido por la agricultura, el aumento es elevado también en industria y servicios, así como entre los inscritos sin actividad económica (jóvenes o parados de larga duración) (Salom y Albertos, 2014, p. 543-545).

Desde el punto de vista de la estructura interna del Área, el efecto de la crisis económica ha sido doble: por un lado, desde una perspectiva territorial, ha tendido a homogeneizar a peor la situación de los distintos municipios tanto en relación con el mercado de trabajo como en cuanto a la capacidad adquisitiva o el índice de actividad económica. Por otro lado, si tenemos en cuenta la distribución de la población, los efectos negativos de la crisis se han concentrado en determinados municipios de la primera corona metropolitana, en particular los ubicados en l'Horta Nord y Sud, aumentando así las desigualdades internas medidas a través del índice de Gini (Salom, J. y Pitarch, M.D., 2014, p. 562-563). Los efectos territoriales de la crisis se han sobreimpuesto a las tendencias seculares de relocalización y descentralización

ISSN: 0212-8594 ISSN-e: 2340-2776.№ DOI: http://dx.doi.org/10.12795/rea.2015.i32.01

REA 32 (2015):1-21

http://editorial.us.es/es/revista-de-estudios-andaluces 
Cuadro 1. Evolución de la tasa de paro

(Paro registrado sobre población potencialmente activa)

\begin{tabular}{|c|c|c|c|}
\hline & PERIODO & $\begin{array}{c}\text { AREA } \\
\text { METROPOLITANA } \\
\text { DE VALENCIA }\end{array}$ & $\begin{array}{l}\text { COMUNIDAD } \\
\text { VALENCIANA }\end{array}$ \\
\hline \multirow{5}{*}{ Tasa de paro } & 1998 & 7,8 & 7,1 \\
\hline & 2001 & 5,2 & 4,2 \\
\hline & 2006 & 5,6 & 5,7 \\
\hline & 2008 & 7,2 & 7,6 \\
\hline & 2011 & 14,6 & 15 \\
\hline \multirow{4}{*}{$\begin{array}{l}\text { Tasa de crecimiento anual } \\
\qquad(\%)\end{array}$} & $1998-2001$ & $-11,41$ & $-13,7$ \\
\hline & 2001-2006 & 1,75 & 7,24 \\
\hline & 2006-2008 & 14,02 & 16,55 \\
\hline & 2008-2011 & 34,4 & 32,58 \\
\hline
\end{tabular}

Fuente: Anuario Económico de La Caixa, varios años.

Cuadro 2. Evolución del paro registrado en el AMV de diciembre de 2007 a diciembre de 2012.

\begin{tabular}{|l|c|}
\hline \multicolumn{1}{|c|}{ SECTOR } & $\begin{array}{c}\text { INDICE } \\
(2007=100)\end{array}$ \\
\hline Agricultura & 335,62 \\
\hline Industria & 260,15 \\
\hline Construcción & 343,20 \\
\hline Servicios & 268,52 \\
\hline Sin actividad económica & 239,45 \\
\hline TOTAL SECTORES & 274,83 \\
\hline
\end{tabular}

Indice 100 (2007=100).

Fuente: Seguridad Social

residencial y de las actividades económicas dando como resultado la modificación de la estructura interna metropolitana.

En el presente artículo presentamos los resultados de un análisis de la estructura socioeconómica interna del Área Metropolitana a fecha de 2011 que, por comparación con la existente en 2001, permite apreciar las tendencias de reestructuración territorial recientes, para, a continuación, y utilizando como patrón de análisis la tipología territorial hallada, estudiar la dinámica reciente de la inversión industrial, una de las variables económicas que ha jugado un papel más importante en la formación del área metropolitana, y que desde distintas instancias viene siendo señalada como un factor estratégico de la recuperación económica. 


\section{LA NUEVA ESTRUCTURA TERRITORIAL DEL ÁREA METROPOLITANA.}

Desde su integridad funcional, el área metropolitana de Valencia ha mostrado históricamente una marcada diferenciación interna en cuanto a usos del suelo y localización de la actividad económica (Sorribes, J. 1997). Esta estructura territorial diferenciada, conformada por los procesos de concentración y descentralización residencial y de las actividades económicas, junto con la inclusión en su ámbito de influencia de áreas próximas articuladas por desarrollos de tipo endógeno o exógeno de carácter más o menos autónomo (sistema productivo del mueble al sur, área industrial de Sagunt al norte), ha experimentado una importante transformación en los últimos años, pasando del clásico modelo radial a una pauta territorial de una mayor complejidad. Los procesos territoriales recientes que han contribuido en mayor medida a la transformación del área metropolitana de Valencia han sido: el fuerte incremento de la urbanización periférica, favorecido por la mejora y ampliación en la oferta de infraestructuras de transporte (Pitarch, M.D. et al. 2010, p.533); el gran incremento de la afluencia de población inmigrante y su desigual distribución territorial (Torres, 2007, p.82-83, p.86), así como el ya comentado impacto de la crisis económica.

La información procedente del Censo de Población y Viviendas de 2011 nos ha permitido, aunque con ciertas precauciones metodológicas y complementado con el Padrón de Habitantes de 2012, comparar dicha estructura con la existente a principios de siglo, definida a partir fundamentalmente de datos del Censo de Población y Viviendas de 2001 completados por información procedente del Padrón de Población del año 2002.

Para ello se ha aplicado, en primer lugar, un análisis de componentes principales en base a 26 variables definitorias de aspectos socieconómicos, demográficos y residenciales. El Análisis de Componentes Principales (ACP) o Factorial permite determinar los factores subyacentes comunes a grupos de variables que se encuentran parcial o totalmente asociadas entre sí. En este caso, se ha aplicado un análisis factorial mediante el método de Componentes Principales $y$, para facilitar la interpretación de los resultados, se ha aplicado una rotación a la matriz de correlaciones mediante el método VARIMAX. El tratamiento estadístico se ha realizado mediante el programa IBM SPSS Statistics versión 22 C. El análisis ha dado resultados significativos de acuerdo con el test de Bartlett (nivel de significación inferior al 0'001 en los dos análisis realizados) y el índice Kaiser-Meyer-Olkin (KMO igual a 0'717 para 2001; y 0’694 en 2011). La selección de los componentes a retener en el análisis se ha hecho a partir de la inspección del gráfico de sedimentación y de las tablas de pesos factoriales (Visauta, B., 1998).

Posteriormente, se ha realizado un agrupamiento cluster a las 89 unidades territoriales que componen el AMV. Los métodos cluster están diseñados para crear grupos homogéneos de casos o entidades y permiten desarrollar una tipología o

ISSN: 0212-8594 ISSN-e: 2340-2776.N№ DOI: http://dx.doi.org/10.12795/rea.2015.i32.01

REA 32 (2015):1-21

http://editorial.us.es/es/revista-de-estudios-andaluces 
clasificación, así como analizar su evolución temporal por comparación entre los distintos periodos de referencia. La estrategia del análisis cluster es buscar la estructura de los datos que no es fácilmente aparente a través de inspección visual (Moreno, P y García, J., 2011). El índice de disimilaridad utilizado ha sido la distancia euclidiana al cuadrado, y el método de agrupamiento, el aglomerativo jerárquico mediante enlace completo, que permite explorar más fácilmente la estructura interna de los datos y es uno de los más rigurosos (un candidato debe tener un cierto nivel de similitud con todos los miembros del grupo), de forma que tiende a formar cluster relativamente compactos compuestos por casos altamente similares, facilitando así la detección de los grupos significativos (Aldenderfer, M.S. y Blashfield, R.K., 1986). Dado que las variables de clasificación proceden del análisis factorial y se encuentran por tanto ya estandarizadas, no se ha considerado necesario aplicar ningún tipo de transformación previa a los datos ni aplicar otros índices de similaridad no afectados por el tamaño que podrían ser más apropiados en otras circunstancias.

La delimitación del área de estudio se ha basado en criterios funcionales, (Salom, J. y Fajardo, F., en prensa) utilizando para ello la delimitación del AMV elaborada por José Ma Feria (Feria, J.M., 2008) basada en un concepto funcional de la ciudad y donde la variable de movilidad residencia-trabajo es el elemento clave para la delimitación de los espacios metropolitanos. De la delimitación original, se han excluido aquellos municipios que, debido a su reducido tamaño demográfico, no presentaban un número mínimo de variables en el año 2011 que permitiera la obtención de resultados significativos en el análisis estadístico realizado. Estos municipios han sido Domeño, Olocau, Petrés, Emperador, y Lloc Nou de la Corona, cuya población sumaba en conjunto 4.187 habitantes en 2011 (0,16\% del total del AMV).

La unidad territorial básica utilizada para el análisis de la estructura interna ha sido mayoritariamente el municipio, debido a las dificultades halladas para obtener información a nivel de distrito censal a partir del Censo de 2011 en los municipios de menor tamaño debido a razones de secreto estadístico. Sólo en el caso del municipio de Valencia ha sido posible tratar la información a escala de distrito censal, lo que parecía recomendable debido al tamaño demográfico del municipio y a la diversidad interna de la propia ciudad. Así, el número final de unidades territoriales analizadas es de 89, 70 municipios y 19 distritos de la ciudad de Valencia.

El análisis de componentes principales ha permitido retener seis factores con autovalores superiores a la unidad, que suman un 76'2\% de la varianza de los datos, y cuya asociación con las variables de análisis aparece en la tabla de pesos factoriales (cuadro 3).

1) El primer componente supone un $24^{\prime} 4 \%$ de la varianza explicada y puede definirse como un indicador del bajo nivel socioeconómico, ya que presenta un alto coeficiente de correlación positiva con la tasa de desempleo, la población con menor nivel educativo, los ocupados en la actividad industrial, y las malas condiciones de la 
vivienda. Por el contrario, presenta correlación negativa con el porcentaje de la población con un mayor nivel educativo y el porcentaje de ocupados en el sector servicios.

2) El segundo componente, con un peso similar al anterior (23'1\% de la varianza explicada), podemos definirlo como representativo de la ciudad consolidada. Presenta altos niveles de asociación con los indicadores relacionados con la antigüedad de la construcción, la población de mayor edad, la presencia de familias unipersonales y la vivienda en régimen de alquiler, y negativos con la población joven, la vivienda hipotecada, y el tamaño medio del hogar. Por el contrario, no presenta ninguna asociación significativa con los indicadores de tipo socieconómico, que quedan básicamente resumidos en el primer factor.

3) Los factores 3 (11\% de la varianza) y 4 (7'5\%), por su parte, aparecen relacionados con el fenómeno migratorio. Mientras que el último está asociado significativamente con la presencia de población inmigrante, el factor 3 es un indicador de la segregación de la inmigración, ya que presenta una correlación positiva con la inmigración procedente de países americanos, y una correlación negativa con la inmigración procedente de países de la Unión Europea.

4) Finalmente, los componentes 5 ( $5^{\prime} 7 \%$ de la varianza explicada) y 6 (4'6\% de la varianza explicada) representan respectivamente las variables sociodemográficas relacionadas con el ciclo de vida y las dinámicas urbanísticas recientes. El componente quinto se encuentra asociado con las características de una demografía expansiva: hogares grandes, con un elevado porcentaje de niños y jóvenes; mientras que el sexto se encuentra asociado a los nuevos crecimientos urbanísticos: presencia de vivienda nueva y en alquiler, y abundancia de población joven.

Cuando comparamos estos resultados con los obtenidos de un análisis paralelo sobre los datos de 2001 (ver cuadro 4), observamos que, mientras que en 2001 es posible alcanzar valores de explicación relativamente elevados ( $70^{\prime} 58 \%$ de la varianza) con sólo los primeros cuatro factores, que han sido identificados respectivamente como centralidad urbana, mala condición socioeconómica, cascos antiguos y nuevos barrios, en 2011 son necesarios hasta 6 factores para alcanzar un nivel similar (76'2\% de la varianza de los datos). Esto se explica por un aumento en la complejidad de la estructura de los datos, lo que significa que la estructura territorial se encuentra afectada por un mayor número de factores significativos, siendo, por tanto, más compleja hoy que a principios de siglo.

Este aumento de complejidad está relacionado con la introducción de dos nuevos componentes relacionados con la inmigración (tercer y cuarto componente en 2011), que no tenían peso propio en 2001, y que han relegado a las variables clásicas de ciclo de vida demográfico y nuevos desarrollos, que en 2001 constituían el cuarto factor significativo en importancia, a las posiciones quinta y sexta. 
Cuadro 3. Matrices factoriales para 2011-2012 con las variables asociadas a los seis primeros componentes principales.

\begin{tabular}{|c|c|c|c|c|c|c|}
\hline \multirow{2}{*}{ VARIABLE } & \multicolumn{6}{|c|}{ PESO FACTORIAL } \\
\hline & FACTOR 1 & FACTOR 2 & FACTOR 3 & FACTOR 4 & FACTOR 5 & FACTOR 6 \\
\hline Población sin estudios & ,828 & &,- 175 &,- 205 & & ,110 \\
\hline Viviendas sin calefacción & ,813 & 197 & ,130 & 109 &,- 272 & \\
\hline Tasa de paro & ,785 & & & ,151 & , 188 & ,179 \\
\hline Población con estudios de primer grado & ,770 & ,166 &,- 140 &,- 180 &,- 179 &, 150 \\
\hline Analfabetos & ,728 & & & & & \\
\hline Extranjeros procedentes de África & ,658 & ,161 & ,245 &,- 186 & &,- 163 \\
\hline Superficie de la vivienda $<90 \mathrm{~m} 2$ &, 588 & &, 584 &, 420 & & \\
\hline Ocupados en la Industria &, 501 &,- 256 &,- 191 &,- 480 & & \\
\hline Ocupados en la Agricultura & ,224 & , 185 &,- 594 &,- 333 &,- 327 & , 175 \\
\hline Población entre 15 y 29 años & , 184 & &,- 125 & ,173 & ,262 &, 613 \\
\hline Vivienda construida entre 1960 y 2001 &, 130 & & ,292 & ,105 & & 809 \\
\hline Población menor de 15 años &,- 126 &,- 765 & &,- 168 & ,233 &,- 132 \\
\hline Hogares con cinco o más personas &,- 149 & & & & ,834 &, 167 \\
\hline Extranjeros procedentes de América &,- 157 &, 113 & ,841 & &,- 166 &, 160 \\
\hline Hogares unipersonales &,- 169 &, 551 &, 113 & ,439 &,- 503 & \\
\hline Extranjeros procedentes de la U.E. &,- 244 &,- 197 &,- 876 & &, 111 & \\
\hline Vivienda en régimen de alquiler &,- 322 & ,316 & ,262 &, 595 &,- 121 & , 192 \\
\hline Ocupados en Servicios &,- 511 & ,150 &, 527 & 491 & ,216 & \\
\hline Viviendas de $120 \mathrm{~m}$. o más &,- 598 &,- 143 &,- 532 &,- 433 & ,228 & \\
\hline Población con estudios de tercer grado &,- 836 & ,205 & ,179 & ,225 & &,- 184 \\
\hline Antigüedad media de la vivienda & &, 839 & ,183 & ,322 & ,106 &,- 162 \\
\hline Mayores de 65 años & & ,838 & &,- 137 & & \\
\hline Vivienda anterior a 1960 & &, 745 & &, 210 & &,- 484 \\
\hline Porcentaje de Extranjeros & & ,204 & & ,821 & &, 179 \\
\hline Tamaño medio del hogar & &,- 477 &,- 123 &,- 420 & ,633 &, 153 \\
\hline Viviendas hipotecadas & &,- 871 & &,- 158 & &,- 319 \\
\hline
\end{tabular}

Fuente: Instituto Nacional de Estadística: Censo 2011 y Padrón 2012. Elaboración propia.

Por su parte, el análisis "cluster" efectuado nos ha permitido definir, tras la inspección del dendograma y el gráfico que representa la relación entre el número de cluster y el coeficiente de fusión, nueve grupos de unidades territoriales, que aparecen representados en el mapa 1.

Sus principales características son las siguientes:

1) Centro y primera corona metropolitana: El núcleo metropolitano aparece organizado en anillos sucesivos a partir de los distritos que constituyen el CBD urbano

- Grupo 1: Está compuesto por los distritos centrales del municipio de Valencia caracterizados por valores positivos en los componentes relativos a la ciudad consolidada, buena situación socioeconómica, e inmigración no comunitaria. Por el contrario, presenta un reducido crecimiento demográfico y urbanístico. Por tanto, este grupo hace referencia a los distritos del casco histórico con viviendas envejecidas y notable presencia de población inmigrante, pero también afectado por un importante proceso de gentrificación.

- Grupo 2: Corresponde a los distritos periféricos de la ciudad de Valencia, situados en torno a los distritos centrales, a los que se unen los municipios de Almussafes, Benisanó y Loriguilla. Estas unidades espaciales presentan características 
Cuadro 4. Matrices factoriales para 2001-2002 con las variables asociadas a los cuatro primeros componentes principales.

\begin{tabular}{|c|c|c|c|c|}
\hline \multirow{2}{*}{ VARIABLE } & \multicolumn{4}{|c|}{ PESO FACTORIAL } \\
\hline & FACTOR 1 & FACTOR 2 & FACTOR 3 & FACTOR 4 \\
\hline Vivienda en régimen de alquiler & ,760 & ,273 & ,268 & \\
\hline Porcentaje de Extranjeros & ,758 & ,242 & &,- 107 \\
\hline Ocupados en Servicios & ,708 & ,177 & ,124 &,- 142 \\
\hline Población con estudios de tercer grado & 696 &,- 103 & ,152 & \\
\hline Hogares unipersonales & , 488 & & ,244 &,- 739 \\
\hline Mayores de 65 años & ,304 & & & \\
\hline Extranjeros procedentes de América & ,270 & 737, & 140, & \\
\hline Tasa de paro & 253 &, 726 & & \\
\hline Hogares con cinco o más personas & ,206 &,- 145 & & ,904 \\
\hline Viviendas de 120 m. o más & 179 &,- 755 & &, 307 \\
\hline Antigüedad media de la vivienda &, 159 &, 167 & 884 &,- 237 \\
\hline Extranjeros procedentes de África &,- 126 & & & \\
\hline Ocupados en la Agricultura &,- 161 &,- 442 &,- 149 & \\
\hline Analfabetos &,- 212 & 258 & & ,191 \\
\hline Tamaño medio del hogar &,- 248 & &,- 220 & ,908 \\
\hline Población sin estudios &,- 281 & &,- 116 & \\
\hline Población con estudios de primer grado &,- 470 & & & \\
\hline Viviendas sin calefacción &,- 476 & ,347 & &,- 222 \\
\hline Población entre 15 y 29 años &,- 526 & ,442 &,- 234 &, 377 \\
\hline Ocupados en la Industria &,- 796 & ,242 & &, 107 \\
\hline Superficie de la vivienda $<90 \mathrm{~m} 2$ & & ,750 & &,- 156 \\
\hline Población menor de 15 años & &,- 160 &,- 391 & ,643 \\
\hline Entranjeros procedentes de la U.E. & &,- 681 & & \\
\hline Vivienda anterior a 1960 & & & ,924 &,- 126 \\
\hline Viviendas hipotecadas & & &,- 508 & \\
\hline Vivienda construida a partir de 1960 & & &,- 949 &, 216 \\
\hline
\end{tabular}

Fuente: Instituto Nacional de Estadística: Censo 2001 y Padrón 2002. Elaboración propia.

socioeconómicas y demográficas similares a las del grupo anterior, si bien con valores medios más bajos. La principal diferencia respecto al grupo anterior es el mayor peso de los componentes relacionados con la inmigración no comunitaria y el crecimiento urbanístico reciente.

- Grupo 3: Constituye, junto con el grupo 4, un segundo anillo en torno a la ciudad de Valencia, al que se une el municipio de Llíria, ubicado en la segunda corona metropolitana. Estas unidades espaciales se caracterizan por concentrar pobreza urbana, altos porcentajes de inmigración (aunque no necesariamente segregada) y valores positivos y moderados de crecimiento demográfico y urbanístico

- Grupo 4: Este grupo está formado por sólo dos unidades territoriales, Albalat de Sorells y distrito 17 de Valencia (Pobles del Nord), que, en comparación con el grupo anterior, presentan un nivel socioeconómico más alto, ausencia de crecimiento urbanístico reciente, y una población más envejecida, lo que parece estar relacionado con su mayor carácter agrario. 
Mapa 1. Tipología de unidades territoriales en el AMV en el año 2011-2012 de acuerdo con el análisis ACP y cluster.

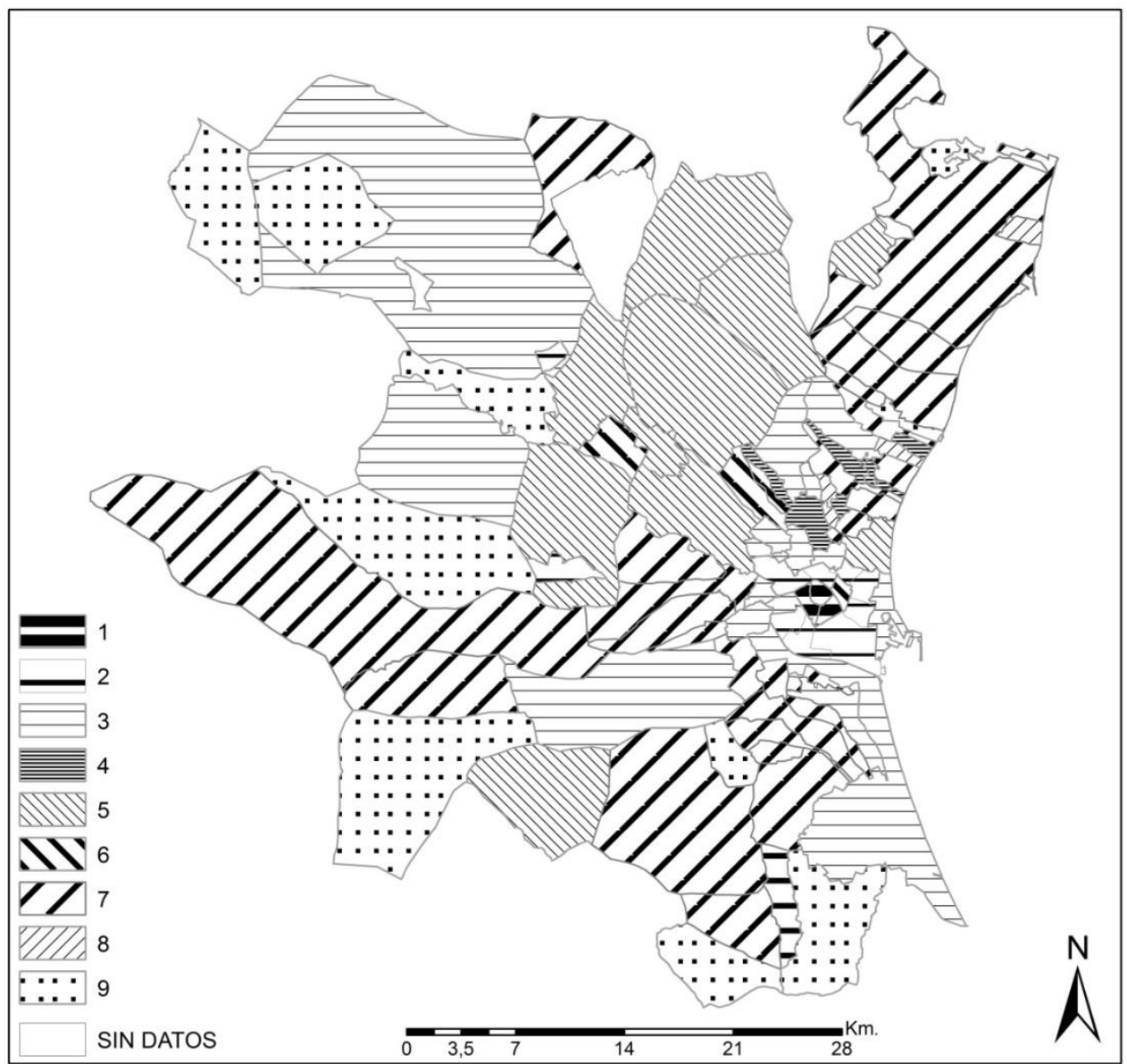

La descripción de los grupos se realiza en el texto.

Fuente: Instituto Nacional de Estadística: Censo 2011 y Padrón 2012. Elaboración propia

2) Segunda corona metropolitana: A diferencia del primer anillo metropolitano, esta segunda corona aparece organizada en sectores, en los que los ejes de comunicación juegan una importante función estructurante.

\section{a) Sector Noroeste:}

- Grupo 5: Aglutina a los municipios situados mayoritariamente en el Noroeste de la segunda corona metropolitana que presentan un nivel socioeconómico elevado, niveles moderados de inmigración de carácter mayoritariamente comunitario, fuerte pujanza demográfica y escaso crecimiento urbanístico reciente.

- Grupo 6: Este grupo puede considerarse como un subgrupo del conjunto anterior. Se encuentra integrado por los municipios de Rocafort, La Eliana y Godella, junto con el distrito 6 (Pla del Real) de Valencia. Presenta características más acentuadas que el

ISSN: 0212-8594 ISSN-e: 2340-2776.N№ DOI: http://dx.doi.org/10.12795/rea.2015.i32.01 
grupo anterior en cuanto a nivel socioeconómico, menor peso de la inmigración, y dinámica demográfica positiva, así como un mayor nivel de consolidación urbana, y -a diferencia del grupo anterior-, un fuerte crecimiento urbanístico.

b) Ejes radiales Norte/Sur-Este/Oeste:

- Grupo 7: Es el grupo que engloba a un mayor número de municipios (28), los cuales se ubican en el eje norte-sur (desde Sagunt hasta Silla) y el eje este-oeste (desde Quart de Poblet hasta Chiva) del AMV. Este grupo se caracteriza por una elevada pobreza urbana, inmigración reducida pero predominantemente no comunitaria, un factor demográfico positivo moderado, y ausencia de crecimiento urbanístico reciente. Además este grupo engloba a aquellos municipios que presentan mayores porcentajes de población ocupada en el sector industrial.

- Grupo 8: Engloba a algunos municipios situados en l'Horta Nord (Bonrepós i Mirambell, Canet, Massalfasar y Vinalesa) que se separan del grupo anterior por su mejor situación económica, y por una menor dinámica demográfica y urbanística. Estos rasgos diferenciales están relacionados con un mayor porcentaje de activos ocupados en la agricultura y mayor presencia de inmigrantes comunitarios.

\section{3) Periferia metropolitana}

Consideramos periferia metropolitana a los municipios constitutivos del grupo 9, que ocupan una posición intersticial entre los ejes transversales antes citados. Se caracterizan por una pobreza urbana elevada, elevado envejecimiento, valores negativos de inmigración, crecimiento urbanístico moderado y una falta de crecimiento demográfico. Estas unidades también presentan mayores porcentajes de activos ocupados en la agricultura que los municipios pertenecientes a los grupos contiguos.

En comparación con la tipología existente en 2001 (figura 2), y sin profundizar en la descripción de los grupos, las principales diferencias apuntan a una profundización en los procesos de segregación funcional dentro del AMV, así como a una ruptura del modelo territorial concéntrico predominante a principios de siglo, que da paso en la segunda corona metropolitana a una estructura territorial más compleja con un mayor peso de los sectores radiales articulados por los principales ejes de comunicación. Estos cambios han afectado fundamentalmente a los municipios ubicados en la segunda corona metropolitana. Mapa 2: Tipología de unidades territoriales en el AMV en el año 2001-2002. 
Mapa 2: Tipología de unidades territoriales en el AMV en el año 2001-2002.

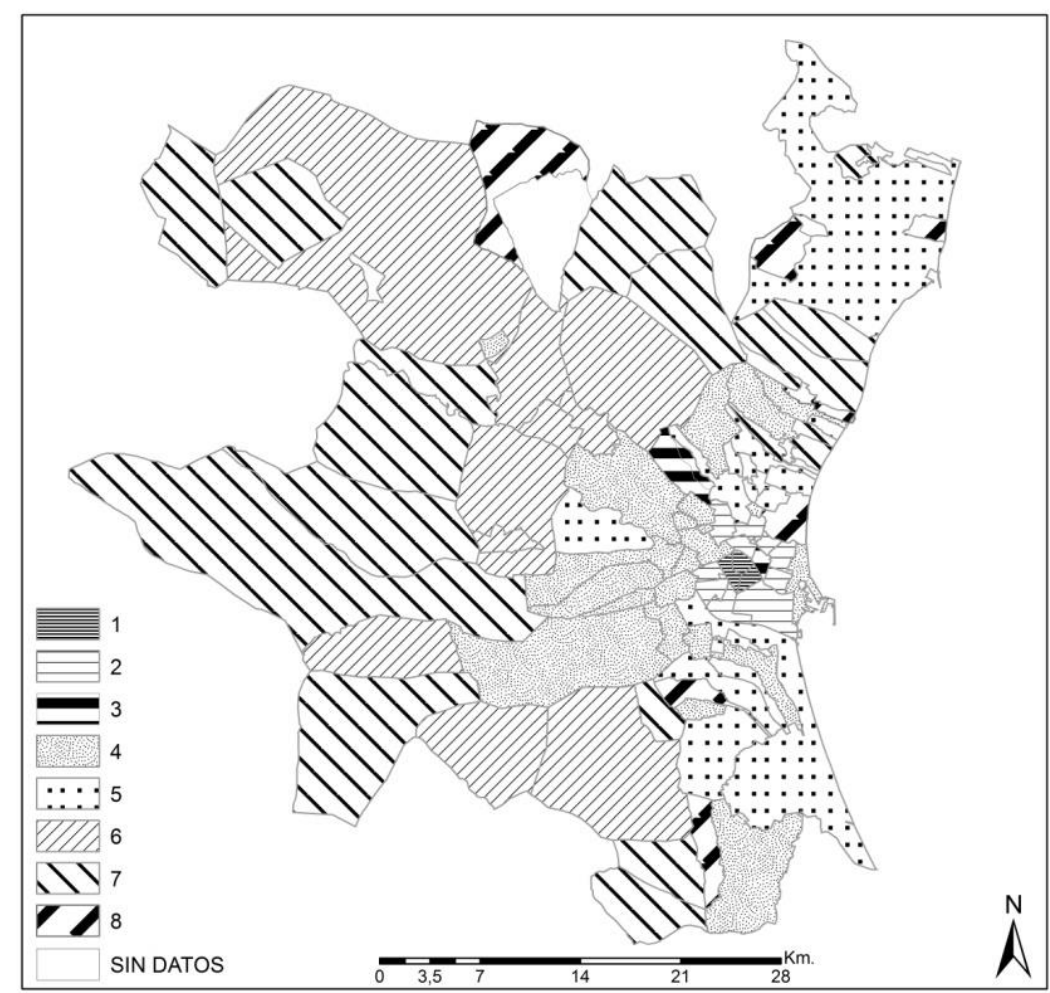

Fuente: Instituto Nacional de Estadística: Censo 2001 y Padrón 2002. Elaboración propia.

\section{CRISIS DE LA INDUSTRIA E INDUSTRIA METROPOLITANA.}

La actividad industrial ha jugado $y$, como hemos visto, sigue jugando un papel fundamental en la articulación del Área Metropolitana de Valencia, de tal forma que tanto el futuro del modelo territorial como las posibilidades de recuperación económica pasan por la dinamización de este sector. Sin embargo, el mal comportamiento de la industria valenciana durante la crisis ha sido uno de los elementos que más ha contribuido al declive económico de la región, con caídas superiores a la media española tanto en valor de la producción, como en empleo y número de empresas. En consecuencia, algunos sectores han quedado muy seriamente afectados, especialmente algunos de los considerados como núcleo de la industria endógena tradicional (mueble, textil, calzado, juguete, y en menor medida, azulejos y plásticos) (Salom, J. y Albertos, J.M., 2014).

Pese a ello, últimamente se han escuchado diversas voces sobre la necesidad de que el crecimiento industrial, -la recuperación de la actividad industrial-, sea una parte fundamental del proceso de salida de la crisis. Este escenario suele plantearse buscando unas bases más sólidas para las economías regionales, compatibles con una mayor y creciente productividad, y capaces de competir en mercados cada vez más globalizados. En este contexto, resulta interesante atender a la evolución reciente de

ISSN: 0212-8594 ISSN-e: 2340-2776.№ DOI: http://dx.doi.org/10.12795/rea.2015.i32.01 
Figura 1: Inversión industrial en la Comunidad Valenciana, 1997-2013.

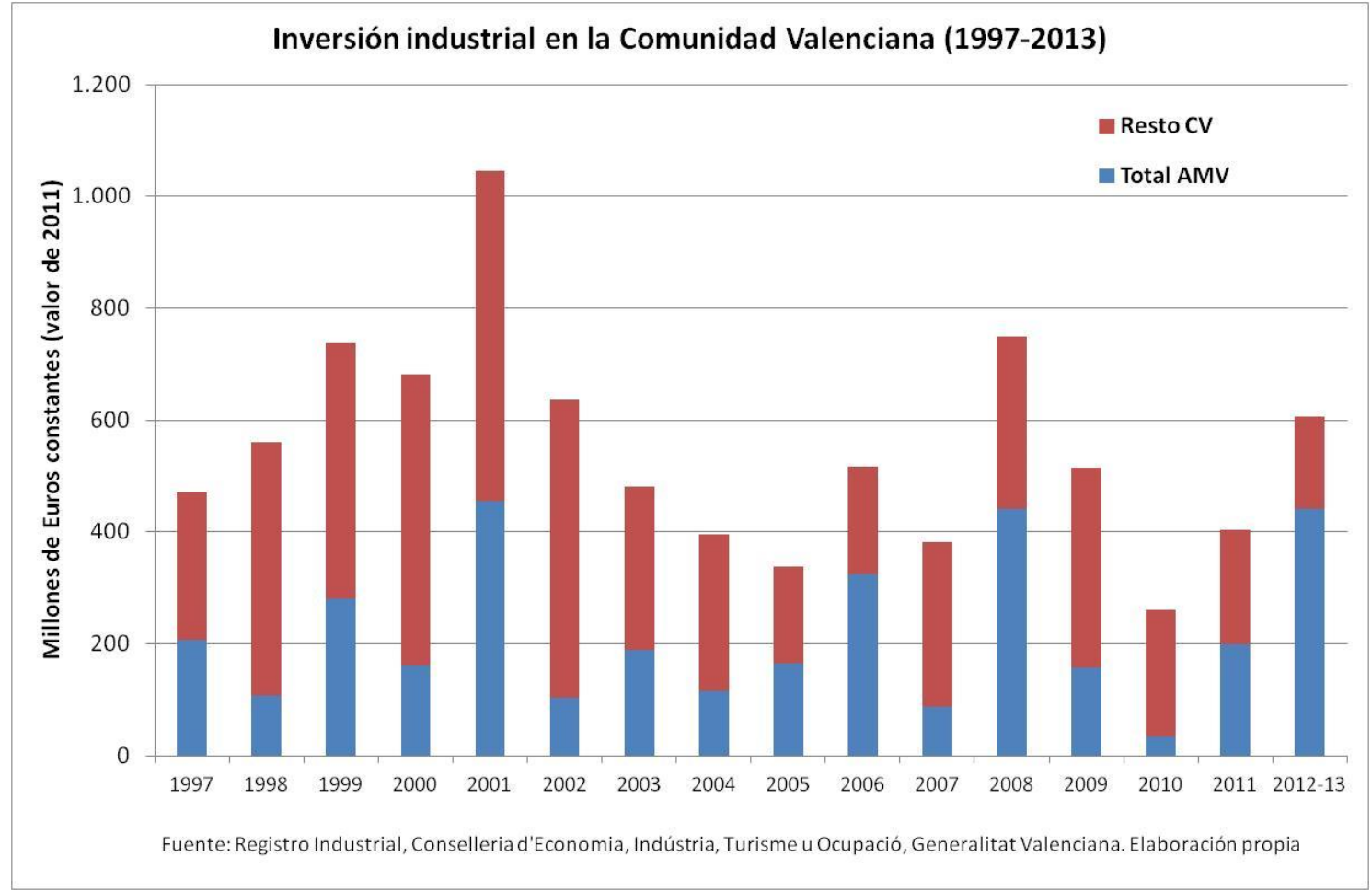

Fuente: Registro Industrial, Conselleria d’Economia, Indústria, Turisme i Ocupació. Generalitat Valenciana. Elaboración propia

la industria con el fin de establecer si este escenario de recuperación industrial es real o factible y, en su caso, cuál sería la dimensión y la forma territorial que -en la escala sub-regional- podría presentar, o está presentando, un proceso de este tipo.

Tal y como muestra la figura 1, la evolución de las inversiones industriales en la región ha tenido un carácter cíclico que se ha desarrollado en paralelo a lo ocurrido en el conjunto de la economía. Tras el final en 2001 del ciclo expansivo que venía teniendo lugar desde mediados de los años 90, se aprecia cómo el modelo de salida de la crisis durante los primeros años del siglo XXI no tiene en la industria un elemento relevante, de forma que las inversiones son decrecientes hasta alcanzar su mínimo en 2005. La recuperación posterior de la inversión industrial no llega a alcanzar los valores propios de la década anterior, y se ve claramente truncada por el estallido de la burbuja inmobiliaria y la depresión posterior a partir de 2008. Así, el año 2010 presenta el mínimo histórico de inversión registrada, debido sobre todo a la práctica inexistencia de inversión industrial en el área metropolitana. No obstante, parece que a partir de este momento la inversión vuelve a mostrar una dinámica creciente, especialmente visible en el bienio 2012-2013. Esta reciente recuperación de la inversión es de componente exclusivamente metropolitana; la inversión industrial en el resto de la región ha seguido cayendo en 2011 y en 2012-2013, hasta suponer sólo el 27\% de la inversión industrial total regional en este último bienio. 
A nivel territorial, cabe destacar que el impacto de la crisis está siendo claramente diferente en los distintos tipos de espacios industriales presentes en la región. En la Comunidad Valenciana el modelo del distrito industrial ha sido el más frecuente a la hora de aglutinar sectores de industria manufacturera, frecuentemente de origen tradicional y endógeno, formando aglomeraciones que territorialmente se organizan en torno a ciudades medias. Junto a este modelo, la industria valenciana también muestra una importante concentración en el Área Metropolitana de Valencia donde, favorecida por economías de urbanización específicas, se han desarrollado junto a algunos sectores tradicionales de iniciativa endógena, otras actividades con mayores exigencias de capital, cualificación y tecnología (industria metálica, química, material de transporte), a menudo con la presencia notable en estos sectores de empresas foráneas o multinacionales.

Así, aunque en los inicios de la presente crisis (2009-2010) la inversión industrial en el ámbito metropolitano se vio afectada de forma más intensa e inmediata que en el conjunto de la región, en el período subsiguiente (2011-2013) es también en este ámbito donde se aprecia más claramente una cierta recuperación. La industria no metropolitana se muestra algo más resistente en el inicio de la crisis, pero a medida que avanza el proceso de deterioro económico, su caída se revela mucho mayor. Este comportamiento diferencial puede tener diferentes explicaciones que será necesario explorar en ulteriores investigaciones. Por un lado, es posible que la estructura sectorial de la industria metropolitana favorezca este mejor comportamiento; por otro, es también posible que el comportamiento diferencial entre industria metropolitana y no metropolitana se derive de otras características estructurales (carácter exógeno vs. endógeno de la iniciativa, grandes empresas multiplanta y/o multinacionales vs. PYMES locales); finalmente, una tercer hipótesis de trabajo sería plantear que las economías de urbanización metropolitanas están siendo más potentes y ventajosas que las economías de localización propias de las aglomeraciones sectoriales características de los distritos industriales.

Más allá del impacto directo de la crisis, es interesante valorar la dispar evolución de la industria y de sus diferentes modelos territoriales a medio largo y plazo. Así, la creciente importancia de la industria metropolitana en detrimento de la localizada en otros espacios regionales, aparece como una tendencia a largo plazo, al menos considerando la evolución seguida en las dos últimas décadas (ver figura 2). Aunque la fuerte variabilidad interanual, especialmente en coyunturas recesivas, de la inversión industrial, enmascara en parte la tendencia subyacente, parece claro que la industria metropolitana se ha mostrado algo más dinámica, especialmente a partir del proceso de salida de la crisis.

Este comportamiento general más positivo de la industria en el AMV presenta diferentes dinámicas a escala intrametropolitana. Como hemos visto, el espacio metropolitano es el producto de un proceso histórico que ha llevado a la formación de un territorio socioeconómica y demográficamente heterogéneo y cambiante. Esta 
Figura 2. Evolución de la inversión industrial en la Comunidad Valenciana y en el Área Metropolitana de Valencia, 1997-2013.

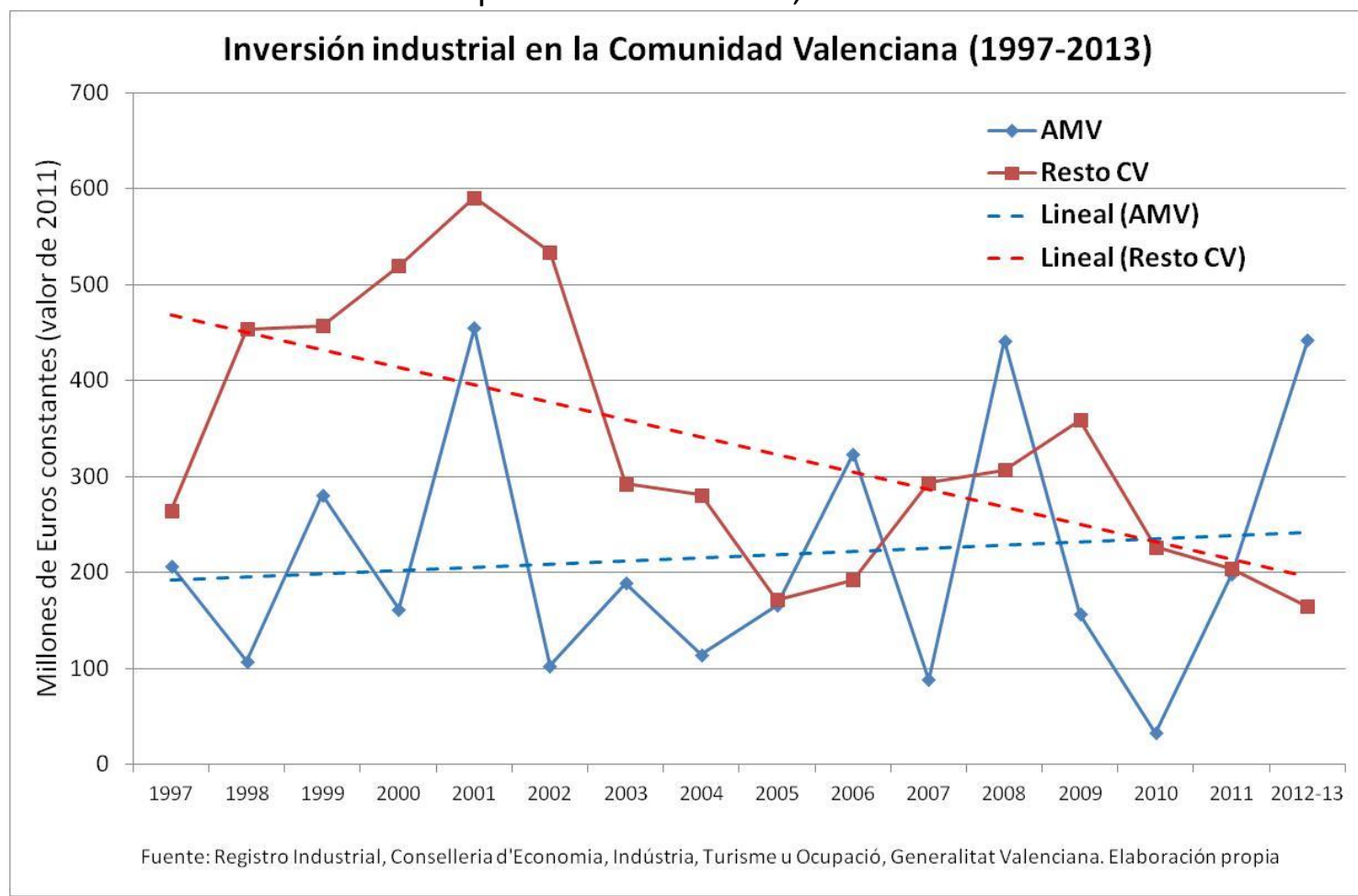

Fuente: Registro Industrial, Conselleria d’Economia, Indústria, Turisme i Ocupació. Generalitat Valenciana. Elaboración propia

división intrametropolitana puede servir como base para el análisis de la inversión industrial, con el fin de enmarcar la dinámica industrial, y especialmente la más reciente, con la crisis, dentro de las estructuras y dinámicas metropolitanas más generales. Para ello, emplearemos la división metropolitana generada a partir del análisis factorial anterior, si bien con algunas pequeñas modificaciones para adaptarla a la especificidad de la información disponible en materia de inversión industrial. La división territorial utilizada es la siguiente (ver mapa 3):

1. Valencia, que se tratará separadamente como núcleo central del área metropolitana aunque con un escaso, prácticamente nulo, peso industrial. Coincide mayoritariamente con las unidades territoriales de los grupos 1 y 2 del análisis factorial.

2. Almussafes, que con la presencia de la principal multinacional en la región (Ford Motor Co.) y su parque de proveedores merece un tratamiento individual.

3. Noroeste residencial (grupos 5 y 6 del análisis factorial), que recoge, entre otros elementos, el grueso de los espacios residenciales suburbanos de la segunda corona que se han desarrollado en el noroeste del área metropolitana.

ISSN: 0212-8594 ISSN-e: 2340-2776.№ DOI: http://dx.doi.org/10.12795/rea.2015.i32.01 
Mapa 3. Delimitación territorial utilizada para el análisis de las inversiones industriales.

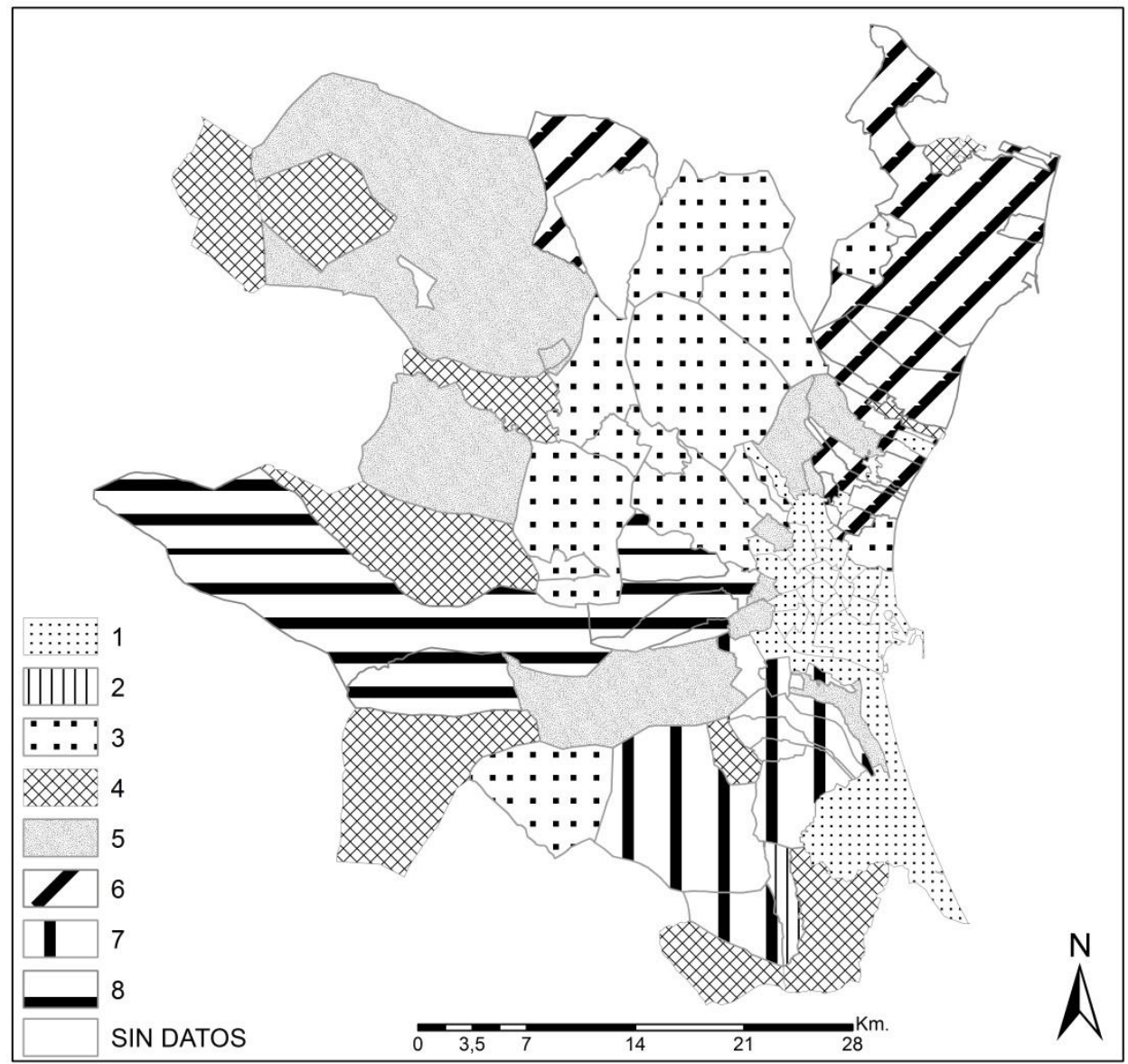

1: Valencia, 2: Almussafes, 3: Noroeste residencial, 4: Municipios periféricos, 5: Espacios conurbados, 6, 7 y 8: Ejes industriales Norte, Sur y Oeste.

4. Periferia metropolitana (grupo 9 del análisis factorial): algunos espacios periféricos del área metropolitana con una mayor componente agrícola y rural.

5. Espacios conurbados con la ciudad de Valencia y otros núcleos con cierto peso industrial de la corona metropolitana (Ribarroja, Torrent, Lliria) (grupos 3 y 4 del ACP)

6, 7 y 8 . Los principales ejes metropolitanos, más allá de la zona conurbada con la ciudad de Valencia, con un desarrollo industrial notable. Corresponden los grupos 7 y $8 \mathrm{ACP}$ que se ha considerado conveniente subdividir para el análisis de las inversiones industriales en eje norte, sur y oeste.

La figura 3 y el cuadro 5 muestran cómo se ha distribuido la inversión industrial dentro del espacio metropolitano tanto durante la actual coyuntura de crisis como en los años anteriores:

ISSN: 0212-8594 ISSN-e: 2340-2776.N№ DOI: http://dx.doi.org/10.12795/rea.2015.i32.01 
- El grueso de la inversión se ha localizado siempre en Almussafes y en los corredores industriales del norte, sur y oeste (Grupo 6-7-8) con valores siempre por encima del $75 \%$ del total metropolitano. Solamente en los inicios de la crisis (2009-10), la caída de inversión en estos espacios fue tan grande que su participación en el total se quedó en el 50\%, una cifra en todo caso reseñable.

- Por el contrario, y en términos generales, los espacios donde la actividad industrial tiene una menor presencia, o donde ésta ha reducido su papel como base económica frente al auge de la función residencial o el crecimiento de las actividades de servicios (Noroeste metropolitano, Valencia y espacios conurbados, periferia metropolitana), la inversión ha ido reduciéndose hasta casi desaparecer.

- En términos generales, la crisis actual, cuyos efectos en la inversión industrial comienzan a ser claramente visibles en 2009, no hace sino agudizar una tendencia anterior de cierto declive industrial. Aunque no de forma tan intensa como en el resto de la Comunidad Valenciana, la industria metropolitana ya experimentaba cierta caída de inversión en el período 2002-2008, cuando en pleno boom inmobiliario la inversión industrial aparecía como una opción poco atractiva. La excepción sería el polo automovilístico de Almussafes, dónde sí se producían inversiones importantes en ese período.

- El punto de inflexión que supuso el impacto inmediato de la crisis (2009-2010) y, sobre todo, la posterior recuperación de la inversión (2011-2013), ha resultado en una clara transformación de estas tendencias. La industria vuelve a registrar cifras de inversión relativamente elevadas a partir de 2011, a la vez que este impulso positivo se concentra como nunca lo estuvo en los espacios más claramente industriales que ahora suman el $92 \%$ del total metropolitano, con un papel muy importante para el polo de Almussafes.

- Se está produciendo, por tanto, una polarización de la localización industrial en el espacio metropolitano como parte de las dinámicas propias introducidas por la crisis, el colapso del anterior modelo de crecimiento, y los patrones espaciales que está siguiendo la parte de la industria que tiene un comportamiento más dinámico.

Para avanzar algo más es preciso considerar un mayor detalle tanto espacial como sectorial, con el fin de caracterizar mejor los elementos más dinámicos de la reciente recuperación de la inversión industrial (2011-2013).

Desde una perspectiva territorial (cuadro 6), el espacio industrial más dinámico se circunscribe al polo de Almussafes y a los ejes norte y oeste del entorno metropolitano (Grupo 1-2-7), mientras que el eje sur no presenta un comportamiento tan positivo. La concentración espacial de la inversión industrial a partir de 2011 es cada vez mayor, de

ISSN: 0212-8594 ISSN-e: 2340-2776.№ DOI: http://dx.doi.org/10.12795/rea.2015.i32.01

REA 32 (2015):1-21

http://editorial.us.es/es/revista-de-estudios-andaluces 
Figura 3. Inversión industrial media anual en el espacio metropolitano. Valores absolutos, 1997-2013.

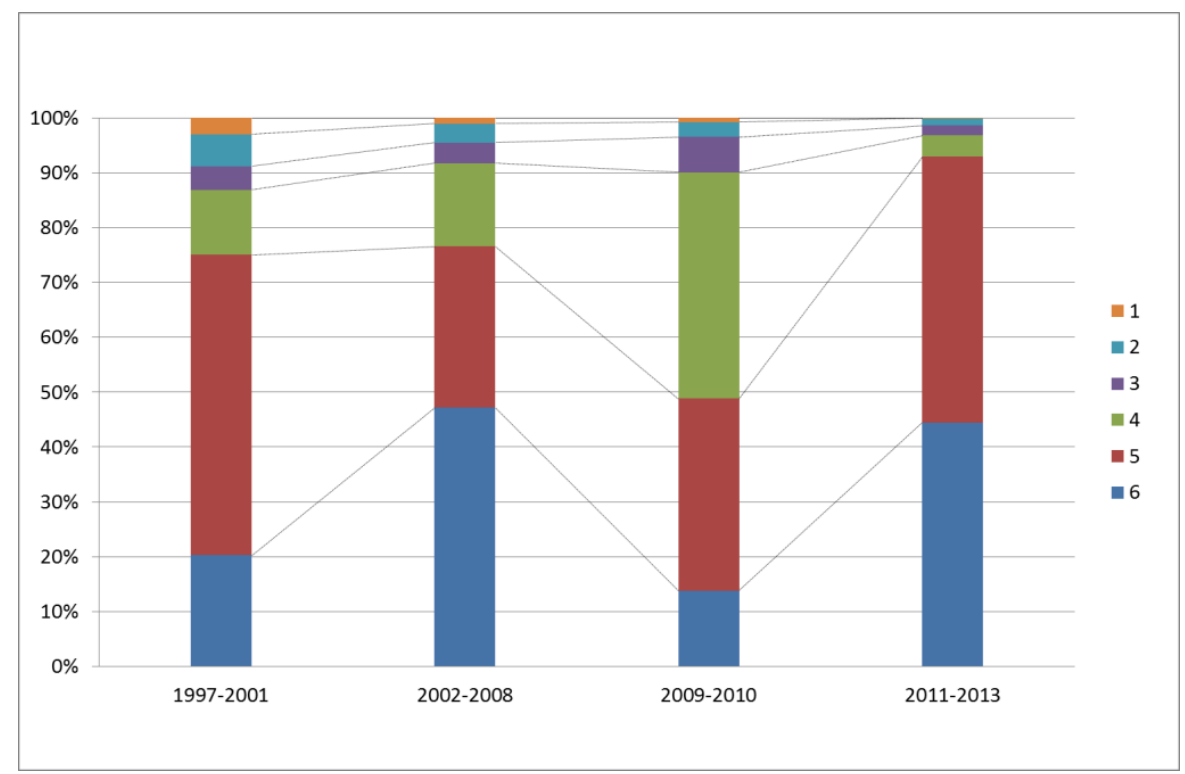

1: Valencia, 2: Espacios conurbados, 3: Periferia metropolitana, 4: Noroeste residencial, 5: Ejes metropolitanos, 6: Almussafes.

Fuente: Registro Industrial, Conselleria d’Economia, Indústria, Turisme i Ocupació. Generalitat Valenciana. Elaboración propia.

Cuadro 5. Distribución de la inversión industrial media anual en el espacio metropolitano. Millones de euros constantes (valor 2011) y porcentaje sobre el total de AMV.

\begin{tabular}{|c|c|c|c|c|c|c|c|c|}
\hline & $\begin{array}{c}1997- \\
2001\end{array}$ & $\%$ & $\begin{array}{c}2002- \\
2008\end{array}$ & $\%$ & $\begin{array}{c}2009- \\
2010\end{array}$ & $\%$ & $\begin{array}{c}2011 \\
-2013\end{array}$ & $\%$ \\
\hline Ejes metropolitanos & 136 & $55 \%$ & 60 & $29 \%$ & 33 & $35 \%$ & 175 & $48 \%$ \\
\hline Noroeste residencial & 30 & $12 \%$ & 31 & $15 \%$ & 40 & $41 \%$ & 15 & $4 \%$ \\
\hline Periferia metropolitana & 11 & $4 \%$ & 8 & $4 \%$ & 6 & $6 \%$ & 7 & $2 \%$ \\
\hline Espacios conurbados & 14 & $6 \%$ & 7 & $4 \%$ & 3 & $3 \%$ & 5 & $1 \%$ \\
\hline Almussafes & 50 & $20 \%$ & 97 & $47 \%$ & 13 & $14 \%$ & 160 & $44 \%$ \\
\hline Valencia & 7 & $3 \%$ & 2 & $1 \%$ & 1 & $1 \%$ & 0 & $0 \%$ \\
\hline $\begin{array}{c}\text { Área Metropolitana } \\
\text { de Valencia }\end{array}$ & 249 & $100 \%$ & 206 & $100 \%$ & 96 & $100 \%$ & 362 & $100 \%$ \\
\hline
\end{tabular}

Fuente: Registro Industrial, Conselleria d'Economia, Generalitat Valenciana. Elaboración propia. 
Cuadro 6. Distribución de la inversión industrial en la crisis en los espacios metropolitanos más dinámicos. Millones de euros constantes (valor 2011) y porcentaje.

\begin{tabular}{|c|c|c|c|c|c|}
\hline & Espacio metropolitano & 2009-2010 & $\%$ & 2011-2013 & $\%$ \\
\hline & Almussafes & 11 & $12 \%$ & 160 & $44 \%$ \\
\hline \multirow{3}{*}{$\begin{array}{l}\frac{\mathscr{d}}{d \frac{\pi}{0}} \\
\frac{0}{0} \\
\frac{\mathbb{U}}{\tilde{U}}\end{array}$} & (Norte) & 12 & $13 \%$ & 97 & $27 \%$ \\
\hline & (Oeste) & 2 & $2 \%$ & 56 & $15 \%$ \\
\hline & (Sur) & 12 & $13 \%$ & 22 & $6 \%$ \\
\hline & Resto del AMV & 57 & $60 \%$ & 26 & $7 \%$ \\
\hline & Total AMV & 95 & $100 \%$ & 361 & $100 \%$ \\
\hline
\end{tabular}

Fuente: Registro Industrial, Conselleria d’Economia, Generalitat Valenciana. Elaboración propia.

forma que los espacios más claramente dinámicos (Almussafes y ejes norte y oeste) reúnen el $86 \%$ de la inversión metropolitana.

A la concentración espacial se une también una notable concentración sectorial y empresarial. Una buena parte de esta inversión responde a unos pocos grandes macroproyectos, en ocasiones ligados a empresas foráneas. Así, ciñéndonos al período que se abre en 2011, casi toda la inversión registrada en Almussafes (el 95\%) corresponde a sólo dos proyectos de inversión en el sector de construcción de vehículos y sus componentes; en el eje norte, el $80 \%$ de toda la inversión corresponde a dos macroproyectos en industria química básica localizados en Sagunt; en el eje oeste se registran algunos grandes proyectos en Quart de Poblet, en los sectores de industria aeronáutica (mantenimiento de aeronaves) y fabricación de bebidas que suman el $83 \%$ de la inversión en este espacio; mientras que en el eje sur un proyecto de inversión en Benifaió en industria alimentaria reúne el $75 \%$ del total de este espacio metropolitano.

En suma, sólo siete macroproyectos industriales (con una inversión media de 126 millones de euros cada uno), localizados en los espacios que aparecen como más dinámicos, suman el $81 \%$ de toda la inversión industrial registrada en el Área Metropolitana de Valencia a partir de 2011. Esta enorme concentración territorial, sectorial y empresarial hace dudar de la solidez de la recuperación emprendida y al no afectar al conjunto del tejido empresarial, explica el escaso impacto que está teniendo en la recuperación del empleo y la producción. Aunque parece claro que el espacio metropolitano es atractivo para la inversión industrial en sectores como el químico, el automóvil o el alimentario, tal y como prueban estas grandes macro-inversiones, la no extensión de estás dinámicas al conjunto del tejido empresarial limita por ahora los impactos positivos que podría tener la industria metropolitana para la salida de la crisis, el incremento general de la actividad y la reducción del desempleo. Estamos, por tanto, ante un entorno de recuperación industrial que, todavía, es poco sólido.

ISSN: 0212-8594 ISSN-e: 2340-2776.№ DOI: http://dx.doi.org/10.12795/rea.2015.i32.01 


\section{CONCLUSIÓN}

Procesos recientes como el aumento de la inmigración y los efectos de la crisis económica parecen haber acelerado los procesos de cambio en la estructura territorial del Área Metropolitana de Valencia, que ha ido evolucionando hacia estructuras más complejas que el modelo radioconcéntrico que la caracterizaba a principios del siglo XXI. El análisis realizado muestra que, tanto en relación con la actividad industrial como con respecto a otras variables socioeconómicas, se está produciendo una diferenciación territorial de carácter funcional cada vez más marcada, que presenta también rasgos de segregación socioeconómica. En el ámbito industrial, una de las fuerzas económicas que más importancia han tenido en la consolidación del AMV tal y como la conocemos hoy, la recuperación de las inversiones en los años 2011-2013 se está produciendo con una notable polarización y concentración tanto espacial como sectorial y empresarial, lo que condiciona tanto en las posibilidades de recuperación económica como el modelo territorial futuro del espacio metropolitano.

\section{BIBLIOGRAFÍA}

Aldenderfer, M.S. y Blashfield, R.K. (1986): Cluster Analysis, Sage University, London.

Feria, J.M. (2008): Un ensayo metodológico de definición de las áreas metropolitanas en España a partir de la variable residencia-trabajo, Investigaciones Geográficas, no 46, pp. 49-68.

Moreno, P. y Castells, M. (2011): Estado del arte en procesos de zonificación, GeoFocus, no 11, p. 155-181

Pitarch, M.D., Albertos, J.M., Cañizares, M.C. (2011): Cambios en la estructura metropolitana durante la última década. El caso del área metropolitana de Valencia, en Gozálvez Pérez, V. y Marco Molina, J.A. (eds.): Urbanismo expansivo. De la teoría a la realidad. XXII Congreso de Geógrafos Españoles, Universidad de Alicante, pp. 527-539.

Salom, J. y Fajardo, F. (en prensa): "La estructura interna del Área Metropolitana de Valencia. Situación actual y evolución reciente (2001-2011) en: Estructura, vida y gobierno en territorios complejos. El área metropolitana de Valencia, ed. Tirant lo Blanch, Valencia.

Salom, J. y Pitarch, M.D. (2014): “El área metropolitana de Valencia en la crisis", en Albertos, J.M. y Sánchez, J.L. (coords.) Geografía de la crisis económica en España, pp.539-563, PUV-Publicaciones de la Universidad de Valencia, Valencia.

ISSN: 0212-8594 ISSN-e: 2340-2776.№ DOI: http://dx.doi.org/10.12795/rea.2015.i32.01

REA 32 (2015):1-21

http://editorial.us.es/es/revista-de-estudios-andaluces 
Salom, J.; Albertos, J.M. (2014): "La crisis económica en los distritos industriales valencianos", en Albertos, J.M. y Sánchez, J.L. (coords.) Geografía de la crisis económica en España, pp.467-495, PUV-Publicaciones de la Universidad de Valencia, Valencia.

Sorribes, J. (1997): Comprendre i gestionar la ciutat: Un assaig d'economia i política urbana, 306 pp., PUV-Publicaciones de la Universidad de Valencia, Valencia.

Torres, F. (2007): Nous veïns a la ciutat. Els inmigrants a València i Russafa, 380 pp. PUV-Publicaciones de la Universidad de Valencia, Valencia.

Visauta, B. (1998): Análisis estadístico con SPSS para Windows, Mc Graw Hill, Madrid. 